\title{
Seven Samples of SBS Galaxies in Selected Fields
}

\author{
S. A. Hakopian and S. K. Balayan \\ Byurakan Astrophysical Observatory (BAO), Byurakan 378433, \\ Armenia. E-mail: susanaha@bao.sci.am
}

\begin{abstract}
The results of follow-up spectroscopy completed for seven samples of SBS galaxies in selected fields are briefly presented.
\end{abstract}

It's well known that the only criterion for selection of the FBS objects (First Byurakan Survey; Markarian 1967) was the UV excess. Together with this, one more criterion has been used for selection of SBS objects (Second Byurakan Survey; Markarian et al. 1983), that is, the presence of emission lines on the low dispersion spectra of the plates of the Second Survey. Almost 1300 objects showing such features have been included in the sample of galaxies of the SBS.

Spectra of Markarian (FBS) galaxies, obtained with higher, intermediate resolution confirmed their supposed nature for the vast majority of them. In contrast, follow-up spectroscopy of SBS galaxies revealed a number of absorption objects, especially at magnitudes close to the limit of the plates at about $19.5^{\mathrm{m}}$.

We studied SBS galaxies in seven selected fields (Hakopian \& Balayan 1998). To complete follow-up spectroscopy for these galaxies, spectral observations of the faint "tails" of the seven samples were carried out with ByuFosc spectrograph (Movsessian 2001) at the 2.6m telescope at the Byurakan Observatory of Armenia, with the Long Slit Spectrograph (LSS, at http://www.sao.ru/ $\sim_{\text {gafan/ }}$ devices/LSS) at the $6 \mathrm{~m}$ telescope of the SAO of Russia. 3-D spectroscopy obtained for a number of galaxies with the Multi Pupil Fiber Spectrograph (MPFS, at http: //www.sao.ru/ $\sim$ gafan/devices/MPFS) at the $6 \mathrm{~m}$ has been used for redshift determination as well as for more detailed study (Hakopian \& Balayan 2001 and references therein).

As a result, seven samples of SBS galaxies in the selected fields have finally been constructed to include only objects with confirmed emission spectra with AGN properties (Sy types, LINERS and of Composite behaviour) and with features of star formation activity in different stages, including BCDG and so on.

Table 1. Data on Selected Samples of SBS Galaxies

\begin{tabular}{llllllll}
\hline Selected field & I & II & III & IV & V & VI & VII \\
\hline N (\%AGN) & $70(7 \%)$ & $42(14 \%)$ & $53(6 \%)$ & $79(10 \%)$ & $58(7 \%)$ & $70(10 \%)$ & $44(20 \%)$ \\
Mark gal & $3(1)$ & $3(1)$ & $5(0)$ & $3(0)$ & $4(0)$ & $4(0)$ & $2(1)$ \\
\hline
\end{tabular}

Current results are illustrated in Fig.1 and summarized in Table 1, the rows of which give: (1) order number of the selected SBS field (see Figure 1); (2) 


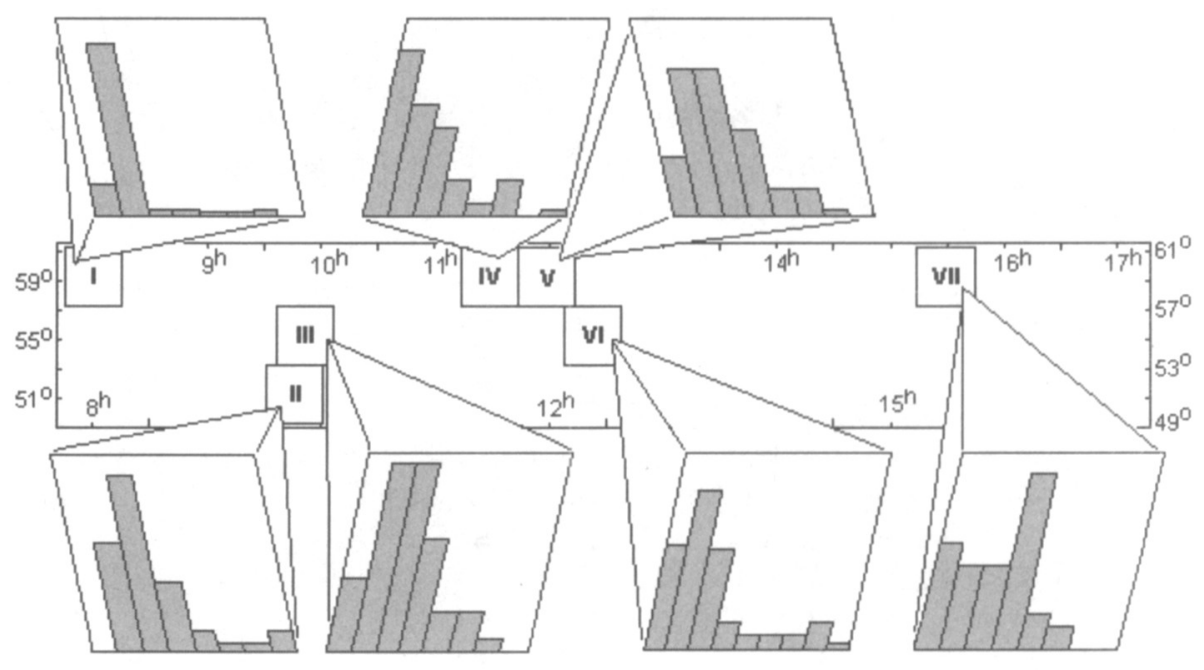

Figure 1. SBS sky region, location of the selected fields and corresponding redshift distribution of SBS galaxies in the fields.

number of galaxies in the sample with the percentage of AGNs in parentheses; (3) number of Markarian galaxies (included in the number given in the previous row) and number of Sy type among them in parentheses.

Figure 1 schematically gives the sky region of the Second Byurakan Survey and the location of the seven selected fields, each of $16 \mathrm{deg}^{2}$. Corresponding redshift distributions in these fields are presented by linked histograms. The range of $\mathrm{z}$ values on the $\mathrm{x}$-axis $0-0.16$, the same in all seven cases, includes the numbers in the samples given in Table 1, except for one or two galaxies in each field with much greater redshifts.

\section{References}

Markarian, B.E. 1967, Astrofizika, 3, 55

Markarian, B.E., Lipovetsky, V.A. \& Stepanian, J.A. 1983, Astrofizika, 19, 29 Hakopian, S. \& Balayan, S. 1999, in "Active Galactic Nuclei and Related Phenomena", Proceedings of IAU Symp. 194, eds. Terzian Y., Weedman D. \& Khachikian E., Astron. Soc. Pacific, Chelsea, Michigan, 162

Movsessian, T., Boulesteix, J., Gach, J.-L. et al. 2001, Baltic Astronomy, 9, 652 Hakopian, S. \& Balayan, S. 2001, Astrophysics (in press) 